\title{
A Decade in the MIST: Learnings from Investigations of Drug Metabolites in Drug Development under the "Metabolites in Safety Testing" Regulatory Guidance
}

\author{
Simone Schadt, Bojan Bister, Swapan K. Chowdhury, Christoph Funk, Cornelis E. C. A. Hop,
} W. Griffith Humphreys, Fumihiko Igarashi, Alexander D. James, Mark Kagan, S. Cyrus Khojasteh, Angus N. R. Nedderman, Chandra Prakash, Frank Runge, Holger Scheible, Douglas K. Spracklin, Piet Swart, Susanna Tse, Josh Yuan, and R. Scott Obach

Roche Pharma Research and Early Development, Pharmaceutical Sciences, Roche Innovation Center Basel, F. Hoffmann-La Roche Ltd., Basel, Switzerland (S.S., C.F.); Boehringer Ingelheim Pharma GmbH \& Co. KG, Biberach a.d. RiB, Germany (B.B., F.R.); Drug Metabolism and Pharmacokinetics Department, Takeda Pharmaceutical International Co., Cambridge, Massachusetts (S.K.C., J.Y.);

Genentech, Inc., Drug Metabolism and Pharmacokinetics, South San Francisco, California (C.E.C.A.H., S.C.K.); Bristol-Myers Squibb Pharmaceutical Co., Princeton, New Jersey (W.G.H.); Research Division, Chugai Pharmaceutical Co., Ltd., Gotemba, Japan (F.I.); PK Sciences (ADME), Novartis Institutes for Biomedical Research, Basel, Switzerland (A.D.J.); PK Sciences (ADME), Novartis Institutes for Biomedical Research, One Health Plaza, East Hanover, New Jersey (M.K.); Unilabs York Bioanalytical Solutions, Discovery Park House, Discovery Park, Sandwich, Kent, United Kingdom (A.N.R.N); Drug Metabolism, Pharmacokinetics and

Clinical Pharmacology, Agios, Cambridge, Massachusetts (C.P.); Merck Biopharma, Quantitative Pharmacology and Drug Disposition, NCE Drug Disposition, Darmstadt, Germany (H.S., P.S.); and Pfizer, Pharmacokinetics, Dynamics and Metabolism, Groton, Connecticut (D.K.S., S.T., R.S.O.)

Received December 6, 2017; accepted February 21, 2018

\begin{abstract}
Since the introduction of metabolites in safety testing (MIST) guidance by the Food and Drug Administration in 2008, major changes have occurred in the experimental methods for the identification and quantification of metabolites, ways to evaluate coverage of metabolites, and the timing of critical clinical and nonclinical studies to generate this information. In this crossindustry review, we discuss how the increased focus on human drug metabolites and their potential contribution to safety and drugdrug interactions has influenced the approaches taken by industry for the identification and quantitation of human drug metabolites. Before the MIST guidance was issued, the method of choice for generating comprehensive metabolite profile was radio chromatography. The MIST guidance increased the focus on human drug metabolites and their potential contribution to safety and drug-drug
\end{abstract}

interactions and led to changes in the practices of drug metabolism scientists. In addition, the guidance suggested that human metabolism studies should also be accelerated, which has led to more frequent determination of human metabolite profiles from multiple ascending-dose clinical studies. Generating a comprehensive and quantitative profile of human metabolites has become a more urgent task. Together with technological advances, these events have led to a general shift of focus toward earlier human metabolism studies using high-resolution mass spectrometry and to a reduction in animal radiolabel absorption/distribution/metabolism/excretion studies. The changes induced by the MIST guidance are highlighted by six case studies included herein, reflecting different stages of implementation of the MIST guidance within the pharmaceutical industry.
Introduction

The study of drug metabolism as applied in the development of new drug candidates was revolutionized by the advent of atmospheric

https://doi.org/10.1124/dmd.117.079848. pressure ionization interfaces for mass spectrometers, which permitted the coupling of high-pressure liquid chromatography (HPLC) to mass spectrometry in the early 1990s. The identification of drug metabolites in complex matrices, such as plasma, became substantially easier. The application of this technology, coupled with the use of radiometric methods, enabled the generation of quantitative metabolite profiles in

ABBREVIATIONS: ADME, absorption/distribution/metabolism/excretion; AMS, accelerator mass spectrometry; AUC, area under the curve; DDI, drug-drug interaction; FDA, Food and Drug Administration; HPLC, high-performance liquid chromatography; HRMS, high-resolution mass spectrometry; ICH, International Conference on Harmonization; ICRP, International Commission on Radiological Protection; MIST, metabolites in safety testing (FDA guidance document); MRM, multiple reaction monitoring; NMR, nuclear magnetic resonance spectroscopy; NOAEL, no observed adverse effect level; P450, cytochrome P450; UPLC, ultraperformance liquid chromatography. 
human plasma, urine, and feces in radiolabel absorption/distribution/ metabolism/excretion (ADME) studies. (In this document, we use the common term ADME to describe this type of study, however, depending on the study design, the information obtained may or may not address absorption or distribution.) The new technology allowed scientists and regulators to step back and examine the role of metabolites in the overall safety evaluation of a drug candidate. Although it was clear that in some instances metabolites could contribute to the pharmacology and toxicology of a drug, and thus should be considered in the safety evaluation, it was less clear what these considerations should look like in practice. Within the industry, the project teams charged with the safe development of new drug candidates faced new dilemmas: Which of these metabolites, if any, are to be considered in risk assessment? Is there a threshold of exposure to a metabolite that makes it important for further investigation? Should the chemical structure of the metabolite be considered in decision-making? Although metabolites are mostly structurally similar to their parent drug, they can technically be considered new chemicals to which humans are exposed. As such, they could theoretically cause safety concerns to human participants in clinical trials unrelated to those potentially caused by the parent drug. Risk assessments are conducted for new drug candidates using a battery of animal and in vitro toxicology studies, but these are routinely done to elucidate the toxicity profiles of the parent drugs and not necessarily for the drug metabolites. Historically, in the absence of knowledge of metabolite profiles across species, assumptions were made that laboratory animal species used in toxicology studies generate the same metabolites as humans. With earlier information on circulating metabolites during clinical development, questions arose regarding the clinical safety of a new drug candidate when there are metabolites that are unique or disproportionate in human relative to toxicology animal species.

Because drug candidate development teams have struggled with the issue of ensuring the safety of human metabolites, a group of scientists from several major pharmaceutical industry research and development organizations, under the auspices of the Pharmaceutical Research and Manufacturers of America, composed a position paper on drug metabolites and the evaluation of their risk (Baillie et al., 2002). In that article, it was stated that metabolites constituting $>25 \%$ of the total drugrelated material in human plasma (typically determined using the HPLC radiometric data from human ADME studies) should also be present in toxicology species at equivalent exposure or greater. Discussions of this issue ensued in various forums and scientific conferences among drug metabolism scientists and toxicologists from industry, regulatory agencies, and academic research institutions. Other articles were published that offered refinements of the original proposal, leveraging examples of toxicities that are known or proposed to be caused by drug metabolites (Hastings et al., 2003; Smith and Obach, 2005, 2006, 2009; Davis-Bruno and Atrakchi, 2006; Ku et al., 2007; Atrakchi, 2009; Leclercq et al., 2009; Walker et al., 2009; Nedderman et al., 2011). A regulatory guidance was first issued in 2008; it decreased the threshold originally proposed by Baillie and colleagues to metabolites comprising $10 \%$ of the parent compound in human plasma (FDA, 2008). Eventually, broader regulatory agreement was reached with the release of guidance from the International Conference on Harmonization (ICH M3(R2); 2009) (ICH, 2010). In this latter guidance, a metabolite constituting $>10 \%$ of the total drug-related material in human plasma at significantly greater levels than the maximum exposure seen in the toxicity studies warrants nonclinical characterization before large-scale clinical trials (usually phase 3 trials) (ICH, 2010, 2013). The FDA aligned with this definition of a disproportionate metabolite in 2016 (FDA, 2016). Although the overarching goal of the guidance is to improve the safe evaluation of a new drug, the more concrete goal is to provide a framework around which companies can devise a strategy around metabolite profiling that should be in line with expectations from regulators. It should be noted that the metabolites in safety testing (MIST) guidance should not be the only focus of metabolite characterization; it is important to note that, in general, the number of circulating metabolites known to cause off-target toxicity is limited, perhaps owing to the difficulty of dissecting properties of parent drug from the metabolite. Nevertheless, the authors of this article have encountered numerous occasions where teams were quick to point to metabolites as the source of unanticipated toxicity, but only in rare cases have such speculations been substantiated with data. It is important to distinguish the presumed toxicity related to stable metabolites and reactive metabolites although both are rare, and few convincing examples exist. The MIST guidance is well qualified to help address issues related to stable metabolites. In some instances, the likely perpetrators of toxicity are chemically reactive metabolites, but they frequently evade detection in vivo, and the only sign of their involvement may be the downstream detection of detoxification products, which renders the MIST guidance of limited help in these situations (Smith and Obach, 2006). An excellent illustration of this type of phenomenon was observed after empagliflozin administration to rat (Taub et al., 2015), where clear evidence for metabolite-mediated toxicity was found, but no direct products of the reactive metabolite were found as circulating species. Finally, pharmacologically active metabolites represent a special class and are frequently worthy of quantitative monitoring, but they are not covered in this article.

In addition to general and genetic toxicities, there has also been a focus on circulating metabolites and their potential to cause or contribute to drug-drug interactions (EMA, 2012; FDA, 2012, 2017). Such cases are relatively infrequent (Yeung et al., 2011; Callegari et al., 2013; Yu and Tweedie, 2013; Yu et al., 2015); however, in one instance, the inhibition of cerivastatin clearance [mediated by CYP2C8 and organic anion transporter (OATP1B1) by the glucuronide metabolite of gemfibrozil led to tragic consequences (Staffa et al., 2002; Shitara et al., 2004). Additional examples include the effect of bupropion metabolites on CYP2D6 levels (Reese et al., 2008) and inhibition of CYP3A4 by desmethyldiltiazem (Rowland Yeo et al., 2010). Regulatory guidance suggests that circulating metabolites present at levels that are a quarter of those of the parent drug (FDA, 2012), and greater than $10 \%$ of drug-related exposure (EMA, 2012) should be evaluated in the standard battery of in vitro $\mathrm{P} 450$ and transporter inhibition assays that are conducted for the parent drug molecule. A new draft guidance was issued by the FDA for public comment (FDA, 2017). In this draft guidance, the FDA suggests taking into account in addition any structural alerts (e.g., for potential time-dependent inhibition). Circulating metabolites at $25 \%$ of parent exposure for metabolites that are less polar than the parent drug and at $100 \%$ of parent exposure for metabolites that are more polar than the parent drug should be evaluated for in vitro drug-drug interaction (DDI) potential according to this draft guidance (FDA, 2017).

Overall, this increased focus on human drug metabolites and their potential contribution to safety and DDIs has led to changes in the practices of drug metabolism scientists responsible for the identification and quantitation of human circulating drug metabolites. Generating a comprehensive and quantitative profile of human metabolites has become a more urgent task, with considerations that are highlighted here as a stepwise approach. Activities such as the definitive human radiolabel ADME study that was usually done late in phase 2 or in phase 3 of clinical drug development (i.e., after demonstration of efficacy) are now of greater focus and may be conducted earlier during clinical development to assess the potential for human metabolites that may be unique or disproportionate. Modern analytical technologies, such as high-resolution mass spectrometry (HRMS), nuclear magnetic 
resonance spectroscopy (NMR), and accelerator mass spectrometry (AMS), are now being used to gather information on human drug metabolites earlier in the drug development timeline. The objective of this cross-industry article is to discuss the changes that have occurred in the practice of drug metabolism science in support of drug development programs that have come about because of the MIST and related guidance documents and the technological advances that have enabled this support. Despite the notion expressed by some that the field of drug metabolism is well established (i.e., a "mature" science), this is hardly the case. This recent focus on drug metabolites, as well as the opportunities afforded by new techniques in the study of drug metabolism, have presented new challenges and made it an exciting time to be in this field.

\section{Brief Discussion of Strategic Proposals Emerging after the MIST Guidance Publication}

Metabolite formation is assessed throughout the drug discovery and development processes; however, different questions are addressed at each stage. The specific metabolite-related strategy for a new molecular entity may differ across drug discovery and development projects and therapeutic targets. It is mentioned in the ICH guidance document that for certain serious therapeutic indications expedited drug development approaches are warranted, and these would be evaluated on a case-bycase basis. The indications mentioned are life-threatening or serious diseases (e.g., advanced cancer, resistant HIV infection, and congenital enzyme deficiency diseases) without current effective therapy ( $\mathrm{ICH}$, 2010, 2013). As for strategy, several overarching factors should be considered at each stage of the development process. The procedures described here allow fit-for-purpose activities to understand more clearly human metabolite coverage in preclinical species. These approaches are compatible with small organic molecule drug candidates and may not cover specific metabolite-related questions for active metabolites, prodrugs, biologics, and antibody drug conjugates.

A typical strategy that drug metabolism scientists follow in support of drug development programs as they address the MIST guidance can be described in three stages.

Stage 1 (Preclinical New Drug). Before the administration of the new compound to humans, drug metabolism scientists are limited to the use of in vitro methods that include human-derived reagents and the use of laboratory animal species. Typically, liver microsomes, liver S9, or hepatocytes are used for this purpose; but, depending on the nature of the metabolites, other matrices such as recombinant enzymes or plasma could be used (Dalvie et al., 2009). Advances in the availability and use of cryopreserved hepatocytes have allowed this model to become commonplace and for researchers to take advantage of the diversity of active drug- metabolizing enzymes contained in these cells. For compounds with very low intrinsic clearance, coculture hepatocyte systems and plateable hepatocytes allow for a much longer incubation time than do cryopreserved hepatocytes in suspension (i.e., days versus hours) (Bonn et al., 2016).

Using these in vitro models, metabolites are generated, and the major metabolites are identified and compared across preclinical species and humans. It is important to note that this comparison is based on chromatographic retention time and mass spectrometry data, whereas the exact structure of most metabolites may not be known. These data are then considered when choosing the appropriate toxicology species. It should be emphasized that these in vitro tools currently have limited predictive power for identifying circulating metabolites (Smith and Dalvie, 2012); however, by using these models to decide on the appropriate toxicology species, the odds for choosing the species that forms the relevant human metabolites are increased.
In addition, circulating metabolites can be qualitatively evaluated in the plasma of laboratory animal species, leading to a better understanding of the predictive power of the in vitro models. Unfortunately, this information may not translate to humans. It is therefore important not to take actions based on preclinical circulatory metabolite information alone. The presence of major circulatory metabolites in a preclinical species greatly increases the odds that those metabolites have coverage in humans.

Stage 2 (Phase 1). During phase 1 clinical studies, plasma samplesusually at steady state from the multiple ascending dose study — can be examined for the presence of circulatory metabolites. With advances in HRMS technology, detection of even minor metabolites has become routine (see later). Although some metabolites might evade detection owing to lack of ionization or large differences in structure and/or MS fragmentation patterns versus the parent compound (rendering MS data unrecognizable compared with the parent drug), examining plasma samples for metabolites has been a relatively reliable method. This is the first opportunity for human circulating metabolites to be identified during the drug development process. In many cases, synthetic standards of metabolites are not available, so the absolute quantification of these metabolites cannot be determined based on the MS response, but sometimes UV data may provide insight as to whether the metabolite is present in high or low abundance relative to the parent drug. The success of using UV data in this manner depends on two things: 1) the plasma concentrations of drug and metabolite being reasonably high and 2) UV absorption characteristics of adequate extinction coefficient at a wavelength greater than $250 \mathrm{~nm}$.

As described in the FDA guidance, a metabolite that exceeds $10 \%$ of the total drug-related material in circulation at steady state requires further evaluation to ensure its presence in the preclinical species used in safety assessments. To determine whether a metabolite reaches the " $10 \%$ trigger," absolute quantities of all the metabolites plus the parent are necessary. This, however, is impossible to achieve at this stage in the absence of radiolabel data or authentic standards of every circulating metabolite. At this stage of the process, absolute quantification may not be required, and apparently prominent human circulatory metabolites could be compared with preclinical plasma samples in a relative fashion (without authentic standards of metabolites). Typically, preclinical plasma samples from toxicology studies at the no observed adverse effect level (NOAEL dose level) are compared with human plasma samples at the anticipated therapeutic dose. For this comparison, typically discrete time-collected pools across all subjects, or area under the curve (AUC) pools (Hamilton et al., 1981; Hop et al., 1998) are prepared. First, relative quantities in human and animal samples are compared using the mixed-matrix method (Gao et al., 2010; Ma et al., 2010; Tong et al., 2010a; Takahashi et al., 2017) to identify potential issues as soon as possible. This method is practical and robust. For the exposure comparison, characterization of metabolite toxicity would generally be considered adequate when animal exposure is at least $50 \%$ of the exposure seen in humans (ICH, 2013); however, when a metabolite constitutes most of the total human exposure, it is appropriate for exposure to the metabolite in animals to exceed that in humans (ICH, 2013).

Rodent radiolabel mass balance studies are often done during phase 1, and when they are conducted, researchers can use the radiolabel response of metabolites that are common between rodent and human as a response factor to quantitate human metabolites (Yu et al., 2007; Xu et al., 2013). In this approach, the HPLC radiometric data for metabolites obtained from profiling rodent biologic matrices (as percentages) are used in combination with the known specific activity of the parent drug to calculate the concentration of each metabolite in that rodent matrix. These data can then be used to calibrate the MS instrument response for 
metabolites of interest, and then metabolite concentrations in human plasma can be measured using this calibrated response. NMR spectroscopy is another technology that can be used for metabolite quantification in the absence of synthetic standards (see later discussion). Both ${ }^{1} \mathrm{H}$ NMR (Dear et al., 2008) and ${ }^{19}$ F NMR (James et al., 2017) have been successfully applied to support projects in development. For fluorinated compounds, this method is very selective since fluorine is not found in endogenous compounds, resulting in low background noise. The major limiting factor for NMR is low sensitivity relative to mass spectrometry.

Comparing the metabolites from plasma samples between preclinical species and humans allows for assessing whether a metabolite has the necessary coverage. If the coverage is adequate, then no further action needs to be taken. If the coverage is not adequate, decisions need to be made about further studies, such as determination of the structure of the metabolite, quantification using an authentic standard, and potentially in vitro and in vivo toxicology studies with the metabolite. Another strategy is to delay further work because it may well be that a metabolite with insufficient coverage turns out to be less than $10 \%$ of the drugrelated material in humans when the radiolabel ADME study is done (see following). Moreover, the economics of drug development are frequently geared toward establishing biologic proof of concept before the conduct of other studies, knowing that failure at the intended indication is the largest source of attrition in drug development (Harrison, 2016). Therefore, additional work on human metabolites may be premature before obtaining clinical proof of concept and the decision to continue with clinical development.

Stage 3. At this stage (by the end of phase 2 and/or before initiation of phase 3), ideally, all the necessary metabolite assessments should be completed. This means quantifying the major human circulating metabolites and comparing them with the metabolites found in preclinical species. This should include the human radiolabel ADME study, which allows full elucidation of the pharmacokinetics and fate of the total drug-related material. Quantitative and comprehensive metabolite profiles in human plasma are obtained by using radiolabeled drug. The human radiolabel ADME study ensures that all metabolites are identified, as well as unknown metabolites that may have been missed by HRMS analysis. In addition, the human radiolabel ADME study identifies which metabolites qualify for DDI assessment, and-even though single dose - can, under certain situations or if samples are pooled appropriately, confirm which metabolites are $>10 \%$ of the total drug-related material in circulation (for MIST) (Prakash et al., 2012). Based on the FDA and ICH guidance, the safety of major human circulatory metabolites must be established before the start of any large clinical study, which in most cases is considered phase 3, although it is worth noting that some phase $2 \mathrm{~b}$ studies may qualify as a "large" study.

Table 1 highlights the typical timing of radiolabel mass balance studies within the companies represented in this review and the timing of discussions of the project-specific MIST strategy with the regulators.

The data gathered in stages 1-3 are leveraged in decision-making by drug development project teams. These include decisions on synthesizing authentic standards of metabolites, developing and using bioanalytical methods to measure metabolites, determining whether more toxicology studies are needed to provide a better risk assessment for a metabolite, testing metabolites for known activities (e.g., activity at target receptor, inhibition of P450 enzymes), and so on. In general, the probability of having to perform a stand-alone in vivo toxicology study with a metabolite is low. As an alternative, performing a toxicology study with an additional species that does form sufficient quantities of the human disproportionate metabolite can be done. In addition, metabolite coverage in different safety studies has different consequences. For example, nonrodent coverage may be fine for general systemic toxicity studies, but it does not provide adequate coverage for reproductive toxicity, genotoxicity, or carcinogenicity. The feasibility of conducting studies with metabolites needs to be considered as well since some metabolites may not have drug-like properties and might not provide adequate exposure after oral administration. In such cases, alternative route of administration, such as intravenous dosing, may need to be considered.

\section{Technical Approaches to Metabolite Profiling and Structure Elucidation: What Has Evolved Since MIST}

The era between 1990 and 2010 was transformative regarding the technology available to detect and identify drug metabolites: the advent of robust commercially available atmospheric pressure ionization mass spectrometers (with tandem quadrupole, ion trap, and time-of-flight detectors) that could be coupled to HPLC, as well as the availability of human-derived in vitro reagents for drug metabolism studies. These tools brought drug metabolism science from an exercise of characterization of drug metabolism profiles in the drug development phase to application in drug design and candidate selection in the drug discovery phase; however, although mass spectrometry offers excellent capability to find drug metabolites in complex biologic matrices, as well as to obtain some preliminary information toward identifying their structures

TABLE 1

Timing of clinical and radiolabel ADME studies within the companies represented in this article and the timing of discussions of the project-specific MIST strategy with the regulators

\begin{tabular}{|c|c|}
\hline \multicolumn{2}{|c|}{ When do you usually conduct radiolabel nonclinical ADME studies? ${ }^{a}$} \\
\hline Preclinical new drug & $27 \%$ \\
\hline Phase 1 & $64 \%$ \\
\hline Phase 2 & $55 \%$ \\
\hline Phase 3 & $0 \%$ \\
\hline \multicolumn{2}{|c|}{ When in clinical development do you usually conduct the human radiolabel ADME study? } \\
\hline End of phase 1 & $0 \%$ \\
\hline Middle of phase 2 & $100 \%$ \\
\hline End of phase 2 & $0 \% /$ \\
\hline Phase 3 & $0 \%$ \\
\hline \multicolumn{2}{|c|}{ When do you usually discuss with regulators how to address a potential MIST issue? } \\
\hline End of phase 1 & $40 \%$ \\
\hline End of phase 2 & $60 \%$ \\
\hline Phase 3 & $0 \%$ \\
\hline
\end{tabular}

${ }^{a}$ More than one answer could be provided. 
(i.e., qualitative information), the quantitation of metabolites by mass spectroscopy requires the availability of authentic standards with which to calibrate instrument response. It is a well known fact that ionization and fragmentation patterns are unique to each new metabolite structure (Hatsis et al., 2017). The ability to detect and qualitatively characterize drug metabolites outpaced the ability to quantitate them. Thus, the use of radiolabeled drugs in generating quantitative metabolite profiles is still necessary, and the human radiolabel ADME study remains the cornerstone of addressing MIST guidance. As this era ended, some considerable technological and methodological advances began to be leveraged in the identification and quantitation of drug metabolites, including HRMS, NMR spectroscopy, AMS for sensitive quantitation of carbon-14, and metabolite biosynthetic methods. Each of these is briefly described as follows in the context of supporting drug development in addressing the MIST guidance.

High-Resolution Mass Spectrometry. At the beginning of the millennium, unit resolution mass instruments were used mainly in drug metabolism; the breakthrough that transformed metabolite profiling was the introduction of rugged and accessible high-resolution instruments with mass accuracy routinely lower than $5 \mathrm{ppm}$. With high resolution, the methods around the use of MS data, along with multiple data mining techniques, quickly became a reality (Zhu et al., 2006; Ruan et al., 2008; Baillie, 2009; Li et al., 2009). These new methods facilitated metabolite detection from complex biologic samples. Low-abundance metabolite ions became more easily found among large complex arrays of ions from endogenous materials in biologic samples and extracts.

The leading HRMS techniques used for metabolite identification today are time-of-flight or Orbitrap mass analyzers with potential for improvement in terms of sensitivity, scan frequency, mass resolution, and mass accuracy. Modern HRMS instruments allow different strategies for metabolite characterization (Zhu et al., 2011), ideally circumventing the multiple-step approach used with nominal mass instruments. The technical improvements on the instrument hardware exert their full power by strong software support; however, major issues remain, such as identification of metabolites resulting from unanticipated and/or novel pathways and distinguishing them from endogenous interferences that are not drug-related, as well as misinterpretation of spectra by automated algorithms. The experience and know-how of the drug metabolism scientists are still required. Multistage mass spectrometry allows analysis of sequential fragmentation reactions, giving insight to the direct relation between product ion and its fragments. In addition, by using the combination of collision-induced dissociation and higher energy collisional dissociation as multistage mass spectrometry, fragmentation affords more comprehensive structural information of putative metabolite(s) (Bushee and Argikar, 2011). HRMS has become the standard mass spectrometry platform for drug metabolite elucidation in recent years.

In addition to qualitative analysis, HRMS can be used to assess metabolite coverage in preclinical species. Ma et al. (2010) have demonstrated that the exposure multiples of human metabolites can be determined in plasma from preclinical species for MIST assessment. In this method, quantitative evaluation of metabolite coverage in preclinical species was performed by mixing equal volumes of appropriately pooled human plasma with blank plasma of animal species and vice versa, followed by an analysis using full-scan HRMS. This approach was shown to provide comparable results to those obtained from regulated bioanalysis and did not require synthetic standards or radiolabeled compounds. In addition, both qualitative and quantitative data could be obtained from a single LC-HRMS analysis on all metabolites. Thus, coverage of any metabolite of interest can be obtained. It should be noted that nominal mass instruments can also be leveraged to accomplish this using multiple reaction monitoring methods (Gao et al., 2010). More recently, Takahashi et al. (2017) reported the determination of exposure multiple of four metabolites and three parent molecules using the same approach and confirmed that the method can reliably be used with an accuracy of $\pm 20 \%$ to that can be achieved using a validated bioanalytical method.

NMR Spectroscopy. A powerful, complementary technique to HRMS is NMR. Given the molecular formula and any structural fragments of complex metabolites as determined from HRMS, NMR, and especially two-dimensional NMR, is invaluable in refining the structural assignment. Since the early 2000s, notable technical innovations have enabled the use of NMR in the field of drug metabolism. Three factors have contributed to this growth: 1) higher magnetic field instruments, 2) the introduction of small volume and cryogenic probes, and 3) new, more elaborate pulse sequences that yield rich structural information (Williams et al., 2016). Cryogenic microprobes have lessened the sample requirements to the point where isolation and characterization of small amounts of metabolites (tens of nanomoles of isolated material), once impossible, is now quite practical. Finally, improved versions of classic experiments, such as the homonuclear correlation spectroscopy, nuclear Overhauser effect spectroscopy, and total correlation spectroscopy, along with heteronuclear experiments like heteronuclear single quantum coherence (direct H-to-C correlations), and heteronuclear multiple bond correlation (multibond $\mathrm{H}$-to-C correlations) experiments offer detailed connectivity data that allows complex structures to be elucidated.

Like hydrogen and carbon, fluorine also has an NMR-active nucleus $\left({ }^{19} \mathrm{~F}\right)$ with sensitivity second only to that of proton NMR. Additionally, ${ }^{19} \mathrm{~F}$ is $100 \%$ naturally abundant, and endogenous levels of fluorine in drug metabolism matrices are nonexistent; these two attributes make it an attractive analyte for pharmaceutical applications (Lindon and Wilson, 2015). Drug metabolism scientists are likely to encounter fluorinated molecules in their studies to which F-NMR can be leveraged.

In the late 1990s, much effort was devoted to coupling liquid chromatography (LC) with NMR for online analysis, in the same way that coupled LC-MS had become the industry standard; however, various technical challenges in the coupling necessitated compromises in both the LC and NMR systems and coupled LC-NMR never achieved the acceptance that LC-MS has found (Walker and O'Connell, 2008). Today, with the advances in NMR sensitivity outlined here, along with numerous systems available to generate more abundant levels of metabolites (see below), the more common practice in pharmaceutical research is to isolate the metabolites of interest by preparative chromatography and run the NMR experiments independently.

The gains in resolution and sensitivity from technical innovations that enabled structural elucidation have also found value for quantitative NMR (qNMR, generally as applied to protons and fluorine) (Webster and Kumar, 2014). NMR can be considered a "universal detector" in that, unlike UV (molar absorptive differences) or MS (ionization efficiencies), protons respond quantitatively in every molecule (presuming relaxation is accounted for). qNMR has been reported on, validated, and accepted from a bioanalytical perspective for purity determinations. More recently, drug metabolism scientists have reported on its application to metabolites (Walker et al., 2014). Isolated metabolites quantitated by NMR can then be used as analytical standards for quantitative MS assays (in vitro and in vivo), as well as tested for activity in various in vitro assays. This information allows for subsequent assessments of contribution of metabolites to pharmacologic activity, quantitative delineation of individual metabolic pathways in drug clearance (with implications for drug-drug interactions), and determinations of metabolite exposure in vivo (MIST).

Given the sensitivity improvements and the quantitative nature of NMR, an obvious application is to measure quantitatively drug 
metabolites in clinical studies in the absence of authentic standards required for MS assays. Indeed, such an approach has been reported in which human plasma metabolites have been detected at levels as low as $10 \mathrm{ng} / \mathrm{ml}$ (Dear et al., 2008); however, the approach has not yet gained broad acceptance, likely because of the high chromatographic threshold required to resolve metabolites from endogenous interferences before quantitation and relatively lower sensitivity than LC-MS. Moreover, NMR equipment is still not very ubiquitous in DMPK departments, which limits its use and impact.

Accelerator Mass Spectrometry. Human mass balance studies are almost exclusively performed with ${ }^{14} \mathrm{C}$-labeled drug. The amount of radiolabeled dose is regulated by the International Commission on Radiological Protection (ICRP) guidance, in which the rat quantitative whole-body autoradiography, sometimes in combination with rat and human pharmacokinetic data, serves as a basis to scale to human tissue exposure and human whole-body dose (ICRP, 1992, 2007). In general, the amount of radioactivity given should not exceed a human wholebody dose of $1 \mathrm{mSv}$ (in many cases, equivalent to a radioactive dose in the order of 50-100 $\mu \mathrm{Ci}$ ), (ICRP, 1991). In some cases, the predicted residence time of the total radioactivity in certain tissues limits the dose of radioactivity that can be given. Under these circumstances, measurement of $\beta$ decay of ${ }^{14} \mathrm{C}$ (using liquid or dry plate scintillation counting techniques) may not be sensitive enough. Today, low levels of ${ }^{14} \mathrm{C}$ can be measured by AMS, a technology that measures the intensity ratio between ${ }^{14} \mathrm{C}$ over ${ }^{12} \mathrm{C}$, and not radioactivity. In human ADME studies that use AMS analysis of ${ }^{14} \mathrm{C}$ levels, doses of less than $1 \mu \mathrm{Ci}$ can be used, with the same clinical dose of total material (i.e., microtracer study; not microdose study.) AMS is based on sample graphitization, followed by combustion and detection of the ${ }^{14} \mathrm{C} /{ }^{12} \mathrm{C}$ ratio. Sample graphitization is a relatively labor-intensive procedure, and recent developments in sample processing have resulted in faster sample turnaround times (van Duijn et al., 2014).

To streamline analysis of human ADME study samples by AMS, various sample pooling strategies are used. In the literature, examples exist where metabolite profiles were generated from a minimum of samples (pooling across subjects), and these were included in a successful submission and registration (Swart et al., 2016; Morcos et al., 2017).

Currently, AMS analysis is carried out in facilities generally separated from the laboratories that are dedicated to metabolite structure elucidation. Smaller benchtop AMS instruments with more facile sample preparation are being developed (Synal et al., 2007; Vuong et al., 2015); with this, microtracer studies could become more regularly used in drug development and could also enable ADME studies to be done earlier in the clinical program, which would yield earlier quantitative metabolite profiles useful for MIST decision-making. Furthermore, the low ${ }^{14} \mathrm{C}$ doses permitted by AMS technology can also be leveraged for more elegant human ADME study designs that include simultaneous intravenous and oral administration for determination of oral absorption and bioavailability, as well as multiple dosing regimens (Graham et al., 2012).

Metabolite Biosynthetic Approaches. With the focus that MIST has brought to metabolites, accompanying efforts to produce metabolites of interest have been on such a scale that they can be readily isolated, quantitated and profiled. Because of the nature of the chemical structures of drug metabolites (i.e., specific stereoisomers and regioisomers, complex conjugates) standard organic synthesis procedures are frequently unable to be readily applied. Whereas technologies such as biomimetic synthesis and electrochemistry have been applied to metabolite synthesis (Cusack et al., 2013), some of the most effective systems for metabolite synthesis are enzyme-based, such as common in vitro reagents (e.g., liver microsomes), engineered P450s, and microorganisms. These systems represent a vast array of synthetic versatility and potential transformations (Trosset and Carbonell, 2015). Biocatalysts offer several advantages over chemical syntheses, often providing better chemo-, regio-, and/or stereo-selectivity under milder conditions; however, relative to other enzyme systems, P450s have poor catalytic efficiency, so numerous efforts have been undertaken to bioengineer catalytic improvements. Behrendorff et al. (2015) have written an excellent review about the evolution of P450s as applied to drug discovery and development). Despite some successes, to date, these efforts have failed to fulfill their potential, as they have failed to achieve the high catalytic rates generally observed in bacterial P450s coupled with the broad substrate acceptance of eukaryotic P450s; current examples typically showcase relatively novel and narrow applications. Clearly, opportunities in this area remain. That said, engineered P450 systems are a useful tool in drug metabolite biosynthesis, and a number of commercial vendors now offer these services.

In contrast to relatively sophisticated techniques associated with bioengineering $\mathrm{P} 450 \mathrm{~s}$, the use of microorganisms to generate metabolites offers relatively simple experimental systems, longer-term stability, generally higher yields of products, and products that are more readily scaled up (Pervaiz et al., 2013; Perkins et al., 2016). One hurdle for the use of bacterial or fungal microorganisms used to generate quantities of a drug metabolite found in animals or humans is that the metabolite derived from the biocatalytic source needs to be explicitly confirmed as structurally identical to that of animals or humans. This can sometimes be a challenge, for example, in the case where the two systems make regioisomeric or stereoisomeric metabolites.

To ensure the production of an authentic metabolite from animals or humans, drug metabolism scientists may use enzyme systems (microsomes, S9, hepatocytes) from the species of interest. Depending on the amount required and the relative turnover, this may be cost-prohibitive; however, advances in qNMR (described above) have made this approach more practical in many cases (Walker et al., 2014). Using this type of approach, metabolites are prepared in sub-micromole quantities, which can be leveraged for bioanalysis since the recovered materials from the NMR analyses are of high enough known concentrations that they can serve as stocks for construction of standard curves needed for HPLC-MS assays. Furthermore, metabolites made in this manner can be used for in vitro assays measuring target and off-target potencies.

Among these four technologies, meeting the needs of the MIST guidelines in modern drug metabolism research demands their application in a fashion complementary to each other; no single approach can accomplish this on its own. If the processes of addressing MIST is viewed in three sequential steps-metabolite 1) detection, 2) structure elucidation, and 3) quantitation - the complementarity of these technologies becomes apparent (Table 2). HRMS offers an excellent capability to detect metabolites, provide some limited structure elucidation, and evaluate metabolite coverage. NMR offers more structure elucidation and can be leveraged as a quantitative tool as well; AMS offers a sensitive quantitative tool; and metabolite biosynthesis technologies offer an ability to generate materials that, when coupled with MS and NMR, can be used by the other techniques for structure elucidation and quantitation.

\section{Case Examples of MIST}

In the following section, selected case studies are presented to highlight technical approaches, challenges, and consequences of MIST assessments. The case studies reflect different stages of implementation of the MIST guidance within the pharmaceutical industry. Case study 1 was conducted before the MIST guidance had been issued and was described in the MIST guidance as case example 2 (FDA, 2016). Case 
TABLE 2

Comparison of four technologies applied in the context of MIST for metabolite profiling and structure elucidation

\begin{tabular}{|c|c|c|c|}
\hline & Detection & Structure Elucidation & Quantitation \\
\hline HRMS & Highly sensitive and effective & Partial information & $\begin{array}{l}\text { Requires authentic standard for absolute quantitation. However, } \\
\text { quantitative coverage assessment can be made without authentic } \\
\text { standard. }\end{array}$ \\
\hline NMR & Not sensitive enough & Highly effective (with biosynthesis) & Quantitative without an authentic standard \\
\hline AMS & Highly effective (requires ${ }^{14} \mathrm{C}$ ) & Not applicable & Quantitative, without an authentic standard \\
\hline Biosynthesis & Not applicable & Highly effective (with NMR) & Can serve as standard for MS quantitation \\
\hline
\end{tabular}

study 2 was conducted around the time of the release of the original FDA MIST guidance in 2008, whereas the others are from a time when the MIST guidelines had been fully implemented by the pharmaceutical industry.

Case Study 1: R483. R483 is a thiazolidinedione peroxisome proliferator-activated receptor $\gamma$ agonist, which was in development for the treatment of type 2 diabetes. It is case example 2 included in the FDA MIST guidance (FDA, 2016). The compound is mainly eliminated by oxidative metabolism via CYP3A4, 2C8, and 2C19, followed by multiple phase 2 conjugations (Bogman et al., 2010).

In vitro metabolite patterns of R483 were qualitatively comparable across species. R483 was more extensively metabolized by human liver microsomes, and the metabolite M4 was the main metabolite in those incubations. In hepatocyte incubations, mostly phase 2 glucuronic acid and sulfate conjugation products of M1 and M2 were observed.

Analysis of early human plasma samples from the single ascending dose study revealed the presence of two borderline/minor metabolites (M1 and M2) and one major metabolite (M4) accounting for greater than
$50 \%$ of total drug-related material based on HPLC-UV (Fig. 1). Subsequently, metabolites were quantified using a validated BA method in subsequent human and animal safety studies to establish their exact abundance in human plasma at steady state and their coverage. The major metabolite (M4) showed an abundance of $\sim 70 \%$ of total drugrelated material, based on validated bioanalytical methods for all known metabolites and was not covered in the two species of animals used for general toxicity studies (rat and cynomolgus monkey). M4 was therefore classified as a major disproportionate metabolite (Fig. 2A), and further safety studies were conducted on this metabolite.

A subchronic toxicity study and an embryo-fetal development study with M4 were conducted in rats. In addition, in vitro genotoxicity testing was conducted for M4, with a positive outcome. Therefore, a subsequent carcinogenicity study was initiated in rats with M4.

Interestingly, analysis of excreta obtained from animal and human radiolabel ADME studies revealed comparable overall metabolism across species. The major human disproportionate plasma metabolite M4 was found in excreta, mostly in feces, across all species with levels
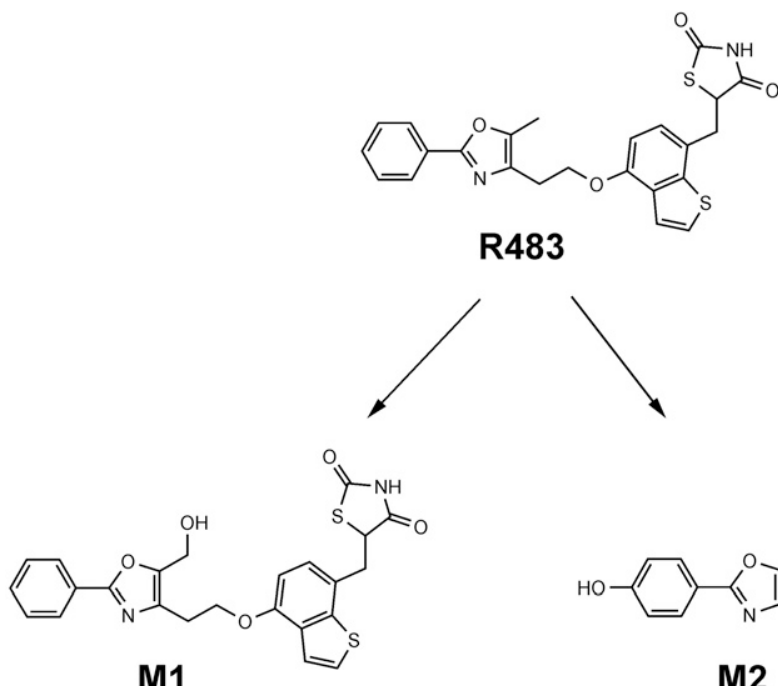<smiles>Cc1oc(-c2ccc(O)cc2)nc1CCOc1ccc(CC2SC(=O)NC2=O)c2sccc12</smiles>

M2

Fig. 1. Metabolic pathways of R483.<smiles>Cc1nc(-c2ccccc2)oc1C(=O)O</smiles> 


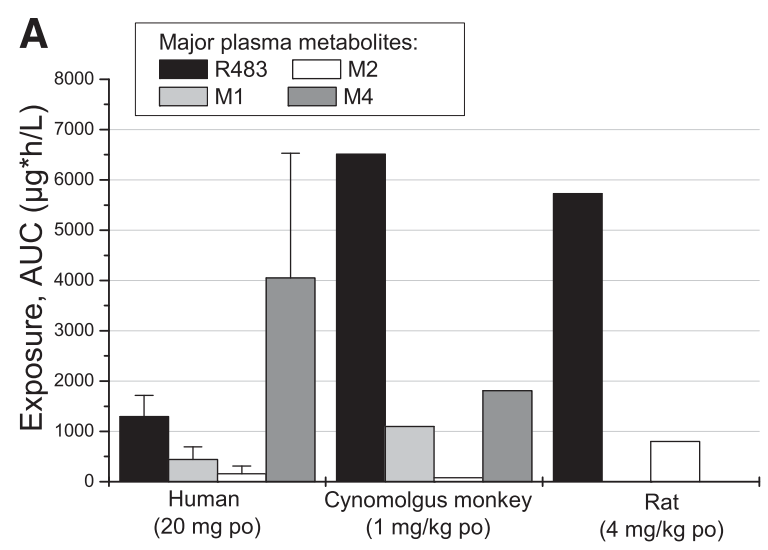

Application of R483 (Dose)

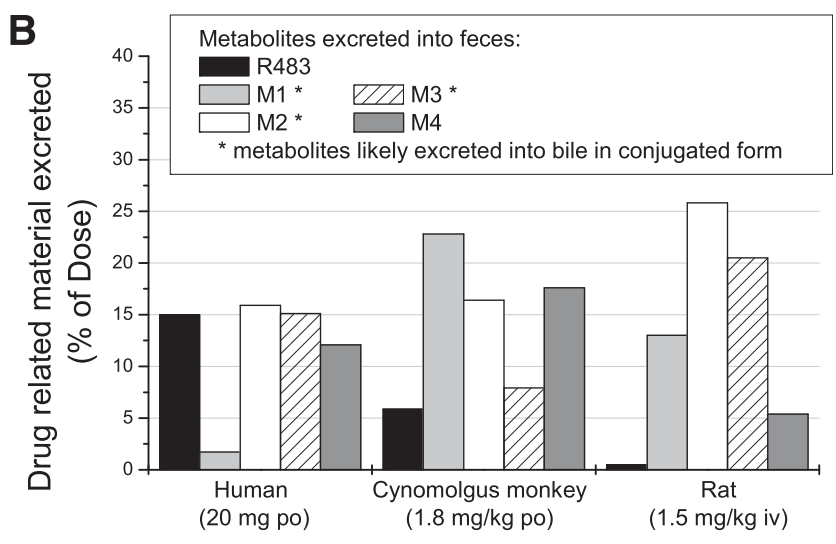

Application of R483 (Dose)

Fig. 2. (A) Systemic exposure of R483 and its metabolites and (B) relative amounts in excreta (\% of dose in feces) across species based on radiolabel ADME data.

of $12.1 \%, 17.6 \%$, and $5.4 \%$ of the dose administered to humans, cynomolgus monkeys, and rats, respectively. A similar picture was observed for the other metabolites (Fig. 2B). Although metabolites were formed in all species (5\%-25\% of the dose in excreta), a striking difference was observed in plasma exposure, particularly for the major human disproportionate metabolite M4.

This case highlights marked quantitative differences in plasma metabolite exposure across species. In contrast to this difference in plasma exposure, the overall metabolism was comparable across species. Therefore, the difference in plasma exposure points toward a striking quantitative difference in metabolite elimination pathways, which are challenging to predict based on in vitro data.

Case Study 2: Vabicaserin. Vabicaserin (PF-05208769, WAY203136, Fig. 3) is a $5 \mathrm{HT}_{2 \mathrm{C}}$ agonist that was evaluated for the treatment of schizophrenia (Dunlop et al., 2011) around the time of the release of the original FDA MIST guidance in 2008 that uses $10 \%$ of parent as the determinant for additional evaluation of metabolites. It is extensively metabolized in animal species (mouse, rat, dog, monkey) and in human both in vitro and in vivo (Tong et al., 2010b). The predominant metabolic pathway in humans was due to carbamoyl glucuronidation; however, other metabolic pathways, including oxidation (with formation of multiple hydroxyl metabolites) and formation of imine and nitrone metabolites, were also observed (Tong et al., 2010a). Metabolite scouting in early clinical trials showed that the carbamoyl glucuronide (M6) was the major circulating entity in plasma, with concentration far exceeding (up to $29 \times$ ) that of the parent as determined quantitatively using validated liquid chromatography-tandem microscopy assay. M6 was also the major entity in human urine, and this metabolic pathway may account for $>50 \%$ of the total clearance based on urinary concentrations (Tong et al., 2010b). Even though formation of M6 was observed in animal species, the systemic exposures to M6 relative to parent appeared to be less than humans (higher amounts in mice, dogs, and monkeys; very low amounts in rats and rabbits), as determined using validated liquid chromatography-tandem microscopy methods. Therefore, the potential existed for M6 being a disproportionate human metabolite. Because of the limited amount of M6 in the systemic circulation in rats and higher amounts in mice, the latter were selected as the rodent species for chronic toxicity studies.

Upon release of the FDA MIST guidance in 2008, it became apparent that other human metabolites formed from the minor pathways might also need to be considered, owing mainly to the fact that the systemic concentration of unchanged vabicaserin was low, and several minor metabolites in systemic circulation would reach the $10 \%$ of parent threshold. As a result, efforts were made to characterize and determine the concentrations of these minor metabolites (to the extent possible) in humans and in animal species. One of the minor metabolites (M5, a nitrone metabolite) was synthesized (Tong et al., 2010a), bioanalytical assays were validated, and sufficient exposure coverage in animals was demonstrated; however, at least two secondary metabolites formed by both hydroxylation and nitrone pathways and three other minor metabolites might also reach or exceed the $10 \%$ threshold relative to parent. Since the synthesis and quantitation of these minor metabolites would be difficult, a type B meeting with the FDA reviewing division was requested to discuss the MIST strategy around vabicaserin.

At the FDA meeting, data were presented to support that toxicity studies in mice and dogs provided adequate coverage for M6 and M5 and no additional studies would be needed. In terms of minor metabolites, the position was presented that a more practical comparison was to use the prominent circulating entity, M6, as the surrogate for the threshold evaluation (10\% relative to M6 rather than to parent) because of the extensive metabolism and low systemic concentrations of the parent compound. This position was in line with, but slightly more conservative than, the threshold of $10 \%$ relative to total circulating material as outlined in ICHM3(R2), which was published after the FDA consultation of vabicaserin was held. Also, because of the difficulty in the synthesis of the secondary metabolites, another proposal was to use materials purified from plasma or urine and determine the concentrations using semiquantitative methods such as NMR, to the extent feasible, if any of these secondary metabolites reached the $10 \%$ threshold relative to M6. Overall, agreements were reached with the reviewing division on the proposed strategy.

This example highlights the overly conservative and potentially burdensome nature of the original FDA MIST guidance, which used $10 \%$ of parent as the trigger for further studies, in cases where the parent compound is extensively metabolized with low systemic concentrations. It should also be noted, however, as stated in the guidance, that the FDA and reviewing divisions encourage sponsors to bring forward

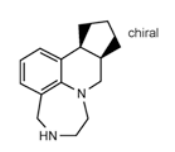

Vabicaserin

Fig. 3. Chemical structure of vabicaserin. 
<smiles></smiles>

azilsartan medoxomil<smiles>CCOc1nc2cccc(C(=O)O)c2n1Cc1ccc(-c2ccccc2-c2noc(=O)[nH]2)cc1</smiles>

\section{azilsartan}<smiles>CCOc1nc2cccc(C(=O)O)c2n1Cc1ccc(-c2ccccc2C(=N)N)cc1</smiles>

M-I

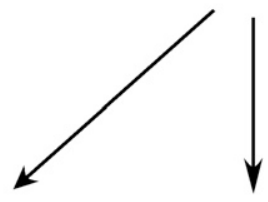<smiles>O=C(O)c1cccc2[nH]c(=O)n(Cc3ccc(-c4ccccc4-c4noc(=O)[nH]4)cc3)c12</smiles>

M-II

Fig. 4. Metabolic pathways of azilsartan medoxomil.

development questions related to metabolites and safety testing, and the agency was open to alternative approaches as demonstrated by this case example. The recent revision of the FDA MIST guidance in November 2016 (FDA, 2016), with an update of the $10 \%$ threshold relative to total drug-related material instead of parent compound, is consistent with ICHM3(R2) and addresses situations such as this case example where the parent compound is extensively metabolized.

Case Study 3: Azilsartan Medoxomil. This case study details the MIST assessment of Azilsartan Medoxomil, which has recently been mentioned also in an article on shared learnings about MIST from an industry and regulatory perspective (Luffer-Atlas and Atrakchi, 2017). Azilsartan medoxomil, a prodrug of azilsartan, is an angiotensin II receptor antagonist for the treatment of hypertension. In both animals and humans, azilsartan medoxomil is rapidly hydrolyzed to azilsartan, the active drug, in the gastrointestinal tract. Azilsartan is further metabolized to two primary metabolites (M-I and M-II; Fig. 4). M-I is formed by decarboxylation, and $\mathrm{M}$-II is formed by $O$-dealkylation (Fig. 4).

In healthy human subjects, most of the radioactivity in plasma was derived from azilsartan (Fig. 5). M-I was a minor human metabolite, accounting for $<1 \%$ of the exposure to azilsartan, whereas M-II was a major human metabolite, accounting for $\sim 55 \%$ of the exposure to azilsartan (31.2\% of the total drug-derived radioactivity).

In rats and dogs (Fig. 6), after oral administration of $1.33 \mathrm{mg} / \mathrm{kg}$ $\left[{ }^{14} \mathrm{C}\right]$ azilsartan medoxomil, most of the radioactivity in plasma was derived from azilsartan, as apparent from the pharmacokinetic profile of total radioactivity and the parent drug. Plasma concentrations of the prodrug azilsartan medoxomil were at or below the limit of quantitation at all time points. Only a small amount of M-I and M-II were present in plasma. In rats, systemic exposures to M-I and M-II were both approximately $2 \%$ of the exposure to azilsartan. In dogs, systemic exposures to M-I and M-II were approximately $0.2 \%$ and $2.4 \%$ of the exposure to azilsartan, respectively. Table 3 summarizes the plasma exposure of M-II, the major human metabolite in rats, dogs, and humans at relevant safety studies and human recommended doses.

Based on these data, it was concluded that the major human plasma metabolite M-II (31.2\% of the total drug-derived radioactivity) was indeed a disproportional metabolite in humans. Subsequently, additional toxicity studies (which included 26-week Tg.rasH2 mouse and 2-year rat studies), including Ames reverse mutation assay, Chinese hamster ovary cell forward mutation assay, mouse lymphoma gene mutation assay, and in vivo mouse and/or rat bone marrow micronucleus assay were conducted by administering metabolite M-II as part of the azilsartan drug development program. In in vivo toxicology studies after administration of M-II, much higher exposure to the metabolite was achieved compared with that in human after administration of azilsartan. These additional toxicity studies were included in the azilsartan medoxomil New Drug Application package, resulting in a successful approval by the US FDA in 2011.

Case Study 4: Venetoclax. Venetoclax is a selective and potent Bcl2 inhibitor $(\mathrm{Ki}<0.01 \mathrm{nM})$ for the treatment of chronic lymphocytic leukemia and other hematologic oncology indications. A human mass balance study was performed using $\left[{ }^{14} \mathrm{C}\right]$-venetoclax (single oral dose of $200 \mathrm{mg}$ ). It was determined that venetoclax was cleared primarily by metabolism, as well as excretion of unabsorbed material. Analysis of pooled plasma $\left(\mathrm{AUC}_{0-48}\right.$ hours $)$ showed that venetoclax was the predominant component at $73 \%$ of the total drug-related material, followed by an oxidative metabolite, M27, at $12 \%$ of the total drugrelated material (Fig. 7); however, radio chromatograms of plasma samples from the mass balance study indicated that the terminal phase elimination half-life of M27 (58.8 hours) was 2.5-fold longer than that of venetoclax; and, at 48 hours and beyond, M27 was the major drugrelated component in the plasma. Examination of mouse and dog plasma (the two toxicology species) showed that M27 was less abundant than in 


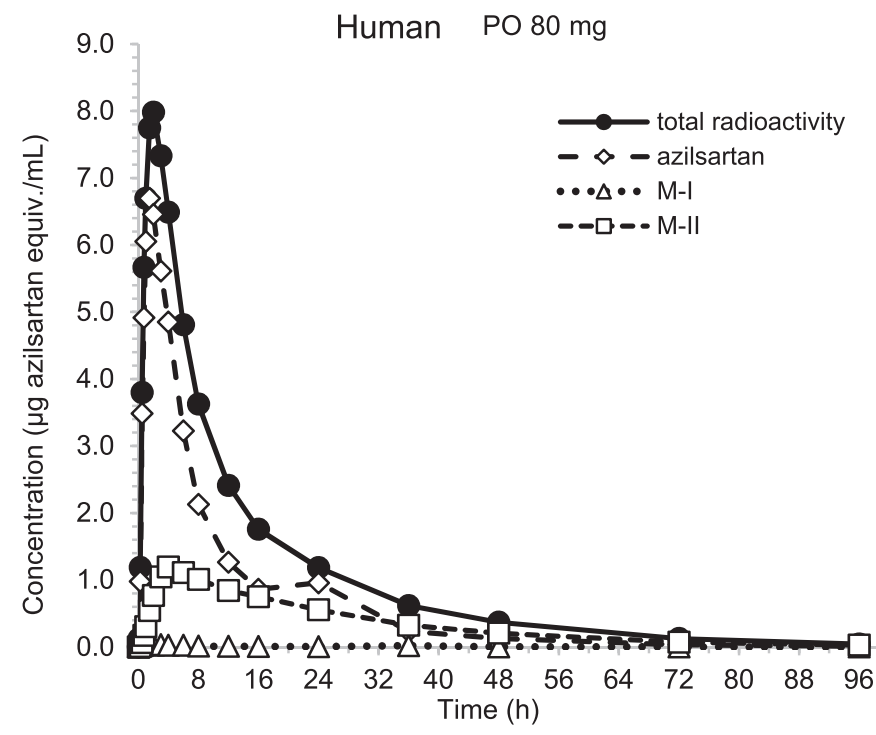

Fig. 5. Mean plasma concentrations of total radioactivity, azilsartan, M-I, and M-II after oral administration of $\left[{ }^{14} \mathrm{C}\right]$ azilsartan medoxomil suspension in healthy male subjects $(n=8)$.

human plasma. The quantitative bioanalytical data using an authentic standard of M27 and a qualified assay showed that the human steadystate AUC of M27 at the therapeutic dose far exceeded the AUCs in the toxicologic species (mouse, dog), confirming that this is a disproportionate human metabolite; however, clinical use involved patients with advanced stage hematologic malignancies, and, therefore, the presence of a disproportionate human metabolite did not hinder or delay further clinical development. Moreover, all clinical safety findings in humans were consistent with the mechanism of action of venetoclax (i.e., inhibition of $\mathrm{Bcl}-2$ ), and the in vitro potency of M27 against Bcl-2 was at least 58-fold less than venetoclax. Therefore, further in vivo safety evaluation was not performed for M27 before its first registration. In addition, M27 was characterized in a secondary pharmacology panel and, from those data, was not expected to have clinically relevant offtarget pharmacologic activity.

The mechanism for formation of M27 was proposed to involve two oxidation steps by CYP3A4, followed by cyclization to form 1,3oxazinane, an uncommon metabolic pathway. It was proposed that the
TABLE 3

Plasma exposure of the disproportional metabolite M-II in rats, dogs and humans

\begin{tabular}{lcc}
\hline \multicolumn{1}{c}{ Species } & Dose & AUC $(0-24 \mathrm{~h}) \mathrm{ng}-\mathrm{h} / \mathrm{ml}^{a}$ \\
\hline Rat (male) & $20 \mathrm{mg} / \mathrm{kg}$ per day & 424 \\
Rat (female) & $200 \mathrm{mg} / \mathrm{kg}$ per day & 1762 \\
Dog (male) & $60 \mathrm{mg} / \mathrm{kg}$ per day & 704 \\
Dog (female) & $12 \mathrm{mg} / \mathrm{kg}$ per day & 188 \\
Human & $80 \mathrm{mg}$ & 22,793 \\
\hline
\end{tabular}

${ }^{a}$ NOAEL doses for rats and dogs and the highest prescribed dose in the human.

first step involves oxidation of the cyclohexene (forming metabolite M5). This metabolite is further o xidized on the piperazine to form an imine and is cyclized by the attack of the hydroxyl group described in the first step, to form a specific stereochemistry of syn-configuration. M27 was not detected in human liver microsomes or recombinant CYP3A4 incubations with venetoclax owing to the low metabolic turnover and being a secondary metabolite; however, M27 was observed in incubations with M5. The latter experiments were done retrospectively after the clinical observation of M27. Its structure was confirmed by comparing the chromatographic retention time and MS/MS spectrum with that of an authentic standard.

Case Study 5: Ribociclib. This case study concerns ribociclib (LEE011), a CDK4/6 inhibitor that was in phase 3 development for the treatment of hormone-receptor positive, human epidermal growth factor-2-negative advanced breast cancer. During the phase 3 trial, clinical trial applications were submitted to two European countries to open additional study centers. At the time these applications were made, the human ADME study results were not available. Exploratory metabolite profiling in human plasma, using samples obtained after a supratherapeutic dose (50\% above the clinical phase 3 dose), was available. In this exploratory in vivo study, 24 metabolites were detected in circulation. Quantification of certain metabolites was achieved using leftover radiolabeled samples from the rat ADME study (Yu et al., 2007). Two metabolites (M13, hydroxylamine; M9, oxidation; Fig. 8)) were expected to be above the MIST threshold.

After regulatory review of the applications, several questions were received. The first health authority asked for information on the comparability of metabolite profiles (in vitro and in vivo) in humans versus animals to support the selection of species used in the toxicology studies. There was a specific request to discuss the toxicologic potential

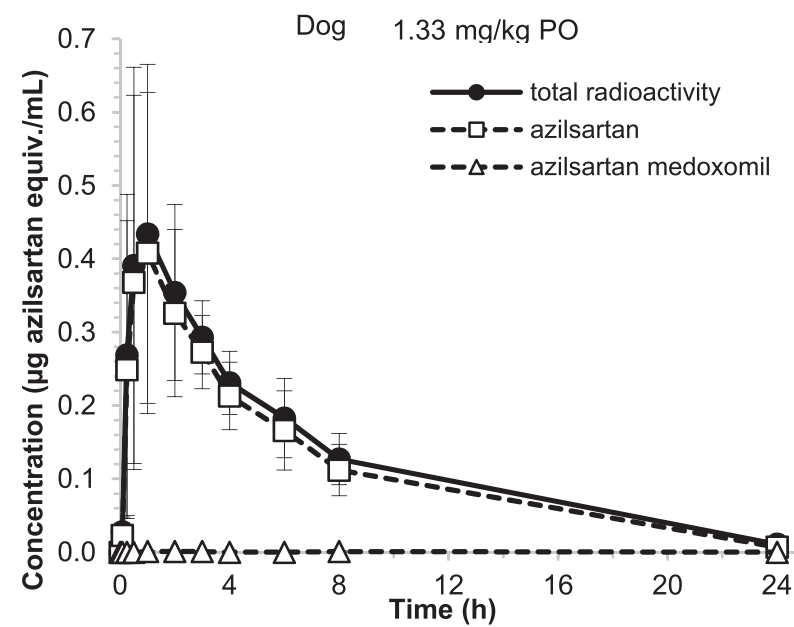

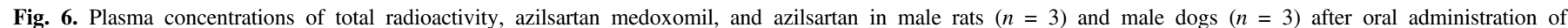
$\left[{ }^{14} \mathrm{C}\right]$ azilsartan medoxomil at $1.33 \mathrm{mg} / \mathrm{kg}$. 


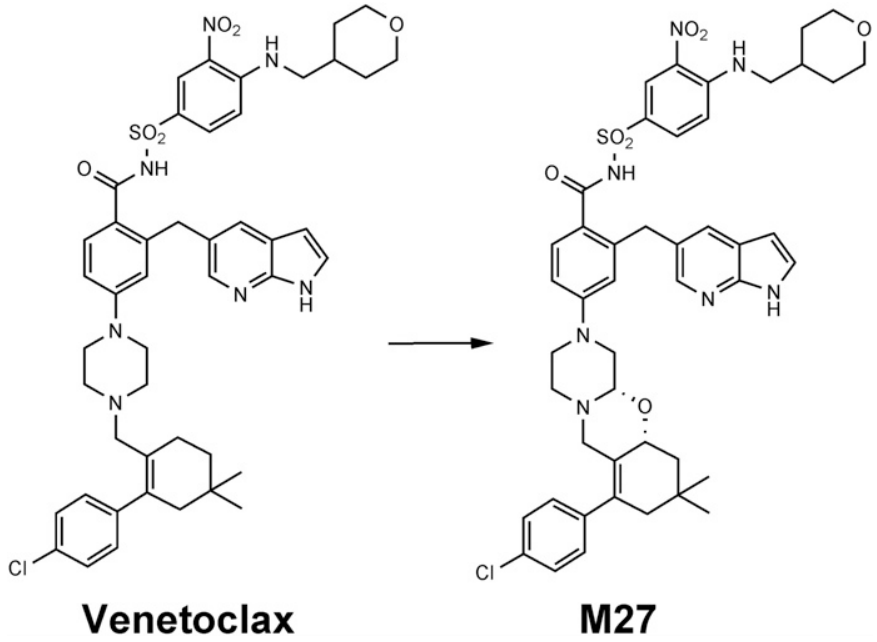

Fig. 7. Chemical structures of venetoclax and its metabolite M27.

of two metabolites (M6, oxidation and glucuronidation; M13, hydroxylamine; Fig. 8), which in vitro were observed only in humans (approximately $1 \%$ of the radioprofile each). The second health authority asked for a comparison of the exposure (AUC) with the parent compound and metabolites, at NOAEL doses, or tolerated doses in animals, to exposures in human at the proposed dose, even though this involved an oncology indication.
A second exploratory metabolite screening study was conducted to estimate animal:human exposure ratios of all identified human circulating metabolites (whether quantitative estimates of exposure were available or not) using a mixed plasma matrix method (based on Gao et al., 2010). The samples used were $\mathrm{AUC}_{0-24 \mathrm{~h}}$ pools (steady state) from 15 -week rat and dog toxicology studies and a single-dose $\mathrm{AUC}_{0-24 \mathrm{~h}}$ pool in human (at a dose $50 \%$ higher than the clinical phase 3 dose). The results of the exploratory studies indicated that M9 and M13, which were the likely MIST-relevant metabolites identified in the first cold study, were covered by male rat with animal:human exposure ratios $\geq 2$. (Metabolite M9 was subsequently found to be below the MIST threshold in the later human ADME study). Metabolite M13 was one of the two highlighted in the health authority questions. The second metabolite of interest, M6, was a glucuronide of a hydroxylated metabolite that was not observed in either rat or dog plasma. The exposure of this metabolite in humans was estimated to be low $(<4 \%$ of observed drug-related material) in the cold study, and no further work was performed. This is in line with the FDA MIST guidance recommendations for phase 2 metabolites that are not acyl glucuronides, where it is stated that phase 2 conjugation reactions generally render a compound more water soluble and pharmacologically inactive, thereby eliminating the need for further evaluation (FDA, 2016). These data were submitted to the health authorities in answer to the questions received. The responses were accepted, and there was no material impact on the development program.

Case Study 6: GDC-0276. For a clinical candidate (GDC-0276; Fig. 9) in development for the treatment of pain by targeting Nav1.7, two major isomeric circulating metabolites (M12 and M13) were detected in

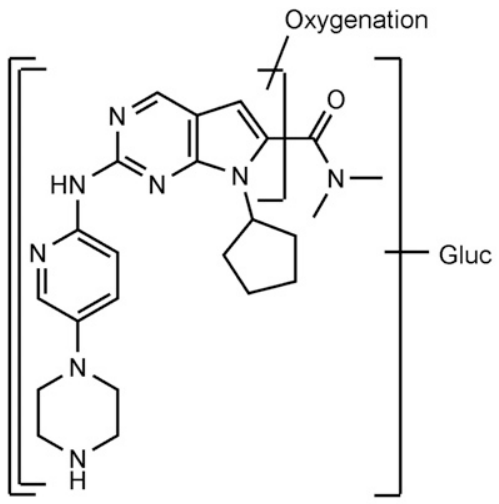

M6

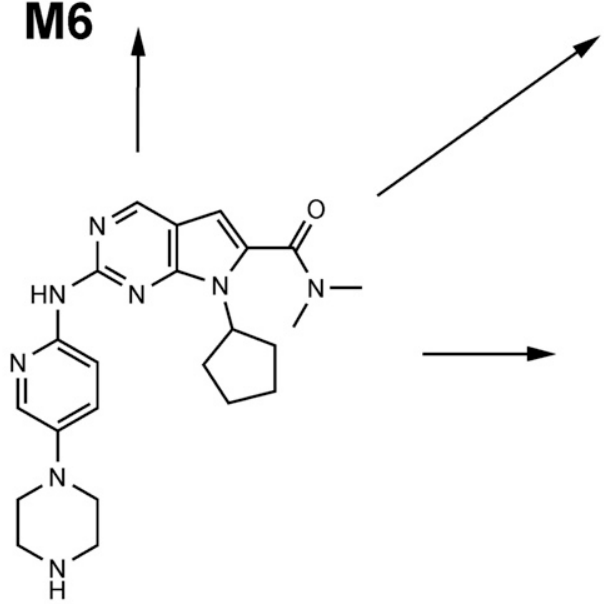

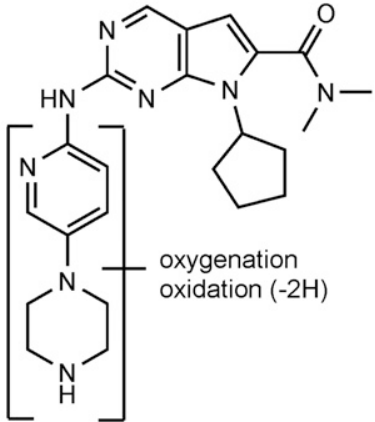

M9<smiles>CN(C)C(=O)c1cc2cnc(Nc3ccc(N4CCN(O)CC4)cn3)nc2n1C1CCCC1</smiles>

Fig. 8. Chemical structures of ribociclib and metabolites discussed in this case study. 
phase 1 dose escalation studies. Both were oxidative metabolites (the addition of one oxygen atom to the adamantyl ring) and were formed by P450 enzymes. The metabolites were synthesized and tested; as expected based on their structures, they were inactive against the target. M12 accounted for greater than 50\% of total drug-related material in circulation based on mass spectrometry and UV response data. Based on the mixed matrix method (Tong et al., 2010a; Ma and Chowdhury, 2011; Takahashi et al., 2017), M12 was disproportionate in human circulation and not covered in rat and dog toxicology species $(<0.1 \%$ preclinical/ clinical exposure), and this was subsequently confirmed by a bioanalytical assay with an authentic standard. M12 was determined to be formed and circulating in rabbit plasma and provided coverage for the embryo fetal development study (seg II). Because of its abundance, M12 was tested alone in a secondary pharmacology panel and an in vivo toxicology study in rats, similar to GDC-0276. M13, on the other hand, was borderline for coverage in dogs and rabbits based on the mixedmatrix method. Furthermore, during the development in phase 1 studies, the recommended phase 2 dose decreased by a factor of 3 , and this changed the coverage of M13 from borderline to solid coverage: from a ratio of 0.9 in dogs at $270 \mathrm{mg}$ twice daily to 2.6 at $90 \mathrm{mg}$ twice daily. In communication with the EMA, the mixed-matrix method was highlighted as appropriate to estimate the relative abundance of M13 in human compared with preclinical species. One important aspect about this assessment was that frontloading studies to monitor metabolites might not be necessary, so a rational tiered approach is more appropriate.

\section{Conclusions}

The MIST guidance grew out of a need from the pharmaceutical industry to define more clearly the expectations of regulatory bodies regarding metabolite profiling. Two factors were driving the industry need: 1) an increased realization that metabolites and metabolic clearance pathways could potentially impact drug efficacy and safety; and 2) advances in analytical technology that provided tremendously enhanced capabilities to generate metabolite data and led to uncertainty as to what level of metabolite characterization was warranted. The two main goals of the regulatory guidance were to 1) improve the overall safety of new drugs and 2) give a framework for metabolite characterization. At the 10-year anniversary of the guidance, it is worthwhile to reflect on our experiences and gain some perspective on how well these goals are being met.

Pharmaceutical research and development organizations have adapted quickly to address the requirements of the MIST guidance. The case studies included herein provide excellent examples of how researchers plan and execute studies to meet the guidance, but it is harder to determine whether there were implications on the safety evaluation of these agents. While it is challenging to determine the

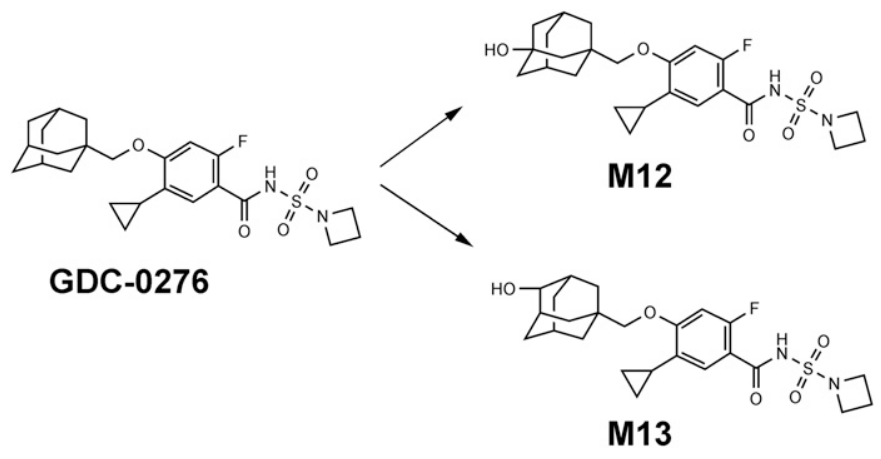

Fig. 9. Structure of GDC-0276 and metabolites M12 and M13. impact of MIST on safety of new drugs, the increased focus on metabolite-mediated safety and how best to investigate it that arose from the guidance and the ensuing literature debate have ensured that metabolite safety issues have become more consistently addressed. It is difficult to find examples where a circulating metabolite, whether the product of a reactive metabolite or not, was thought to be directly linked to a safety signal. We are not aware of any examples of development-stage drugs in the post-MIST era where exposure of a circulating reactive metabolite or a downstream product (e.g., a glutathione or cysteine conjugate) of such a metabolite has been carefully qualified between human and toxicology species. Stable downstream products are typically eliminated rapidly from the body via bile and/or urine resulting from their high molecular weight and hydrophilicity and generally do not circulate; therefore, it is difficult to compare the exposure of reactive metabolites across species in vivo with respect to MIST. Although determination of circulating metabolite levels in human and toxicology species is an important part of overall safety evaluation, determination of metabolites in particular tissues of interest may be more important but obviously not practical in humans. Regarding the general topic of the formation of reactive metabolites, it is worth noting that at about the same time as the MIST guidance was issued, most pharmaceutical companies instituted in vitro screens to minimize the formation of reactive species from their new drug candidates (Gan et al., 2005; Prakash et al., 2008; Brink et al., 2017). The second goal of the guidelines was to provide expectations for metabolite profiling that serve as a framework for discussions between regulatory authorities and pharmaceutical researchers. In this respect, we agree that discussions with regulatory bodies have become more straightforward and less contentious on the issue of metabolite characterization.

Before the MIST guidance was issued, the method of choice for generating comprehensive metabolite profiles was radiochromatography. Typically, in vitro metabolite profiles of human and animals and in vivo metabolite profiles of animals were conducted before phase 1, and analysis of human samples was almost always done in the context of a human ADME study, usually late in phase 2 or in phase 3 of clinical drug development. The MIST guidance increased the focus on human drug metabolites and their potential contribution to safety and drug-drug interactions and led to changes in the practices of drug metabolism scientists. Generating a comprehensive and quantitative profile of human metabolites has become a more urgent task. This, together with technological advances, led to a general shift of the focus toward early human studies using high-resolution mass spectrometry and to a reduction in animal studies using radioactivity.

Overall, the guidance documents produced by the regulatory authorities have clearly had a significant impact on the conduct of metabolite safety assessments within the pharmaceutical industry. Although the specific scientific approaches have largely been defined by experts from within the drug metabolism and pharmacokinetics community, the MIST guidelines, together with the preceding seminal publications from leading industry scientists and the ensuing literature debate, have resulted in more consistent thinking about the purpose and conduct of metabolite characterization studies in support of drug safety. As a result, considerations of metabolite safety are more firmly embedded in the minds of metabolism scientists, such that the safety profiles of novel compounds are more thoroughly and consistently supported.

\section{Authorship Contributions}

Wrote or contributed to the writing of the manuscript: Schadt, Bister, Chowdhury, Funk, Hop, Humphreys, Igarashi, James, Kagan, Khojasteh, Nedderman, Prakash, Runge, Scheible, Spracklin, Swart, Tse, Yuan, Obach. 


\section{References}

Atrakchi AH (2009) Interpretation and considerations on the safety evaluation of human drug metabolites. Chem Res Toxicol 22:1217-1220.

Baillie TA (2009) Approaches to the assessment of stable and chemically reactive drug metabolites in early clinical trials. Chem Res Toxicol 22:263-266.

Baillie TA, Cayen MN, Fouda H, Gerson RJ, Green JD, Grossman SJ, Klunk LJ, LeBlanc B, Perkins DG, and Shipley LA (2002) Drug metabolites in safety testing. Toxicol Appl Pharmacol 182:188-196.

Behrendorff JB, Huang W, and Gillam EM (2015) Directed evolution of cytochrome P450 enzymes for biocatalysis: exploiting the catalytic versatility of enzymes with relaxed substrate specificity. Biochem J 467:1-15.

Bogman K, Silkey M, Chan SP, Tomlinson B, and Weber C (2010) Influence of CYP2C19 genotype on the pharmacokinetics of R483, a CYP2C19 substrate, in healthy subjects and type 2 diabetes patients. Eur J Clin Pharmacol 66:1005-1015.

Bonn B, Svanberg P, Janefeldt A, Hultman Ia, and Grime K (2016) Determination of human hepatocyte intrinsic clearance for slowly metabolized compounds: comparison of a primary hepatocyte/stromal cell co-culture with plated primary hepatocytes and HepaRG. Drug Metab Dispos 44:527-533.

Brink A, Pähler A, Funk C, Schuler F, and Schadt S (2017) Minimizing the risk of chemically reactive metabolite formation of new drug candidates: implications for preclinical drug design. Drug Discov Today 22:751-756.

Bushee JL and Argikar UA (2011) An experimental approach to enhance precursor ion fragmentation for metabolite identification studies: application of dual collision cells in an orbita trap. Rapid Commun Mass Spectrom 25:1356-1362.

Callegari E, Kalgutkar AS, Leung L, Obach RS, Plowchalk DR, and Tse S (2013) Drug metabolites as cytochrome $\mathrm{p} 450$ inhibitors: a retrospective analysis and proposed algorithm for evaluation of the pharmacokinetic interaction potential of metabolites in drug discovery and development. Drug Metab Dispos 41:2047-2055.

Cusack KP, Koolman HF, Lange UE, Peltier HM, Piel I, and Vasudevan A (2013) Emerging technologies for metabolite generation and structural diversification. Bioorg Med Chem Lett 23: 5471-5483.

Dalvie D, Obach RS, Kang P, Prakash C, Loi CM, Hurst S, Nedderman A, Goulet L, Smith E, Bu HZ, et al. (2009) Assessment of three human in vitro systems in the generation of major human excretory and circulating metabolites. Chem Res Toxicol 22:357-368.

Davis-Bruno KL and Atrakchi A (2006) A regulatory perspective on issues and approaches in characterizing human metabolites. Chem Res Toxicol 19:1561-1563.

Dear GJ, Roberts AD, Beaumont C, and North SE (2008) Evaluation of preparative high performance liquid chromatography and cryoprobe-nuclear magnetic resonance spectroscopy for the early quantitative estimation of drug metabolites in human plasma. J Chromatogr B Analyt Technol Biomed Life Sci 876:182-190.

Dunlop J, Watts SW, Barrett JE, Coupet J, Harrison B, Mazandarani H, Nawoschik S, Pangalos MN, Ramamoorthy S, Schechter L, et al. (2011) Characterization of vabicaserin (SCA-136), selective 5-hydroxytryptamine 2C receptor agonist. J Pharmacol Exp Ther 337:673-680.

European Medicines Association (EMA) (2012) Guideline on the Investigation of Drug Interactions.

Food and Drug Administration (FDA) (2008) Guidance for Industry: Safety Testing of Drug Metabolites, US Department of Health and Human Services FaDA, Center for Drug Evaluation and Research, Silver Spring, MD.

FDA (2012) Guidance for Industry: Drug Interaction Studies - Study Design, Data Analysis, Implications for Dosing, and Labeling Recommendations, US Department of Health and Human Services FaDA, Center for Drug Evaluation and Research, Silver Spring, MD.

FDA (2016) Guidance for Industry: Safety Testing of Drug Metabolites, US Department of Health and Human Services FaDA, Center for Drug Evaluation and Research, Silver Spring, MD.

FDA (2017) Draft Guidance for Industry: In Vitro Metabolism and Transporter-Mediated DrugDrug Interaction Studies, US Department of Health and Human Services FaDA, Center for Drug Evaluation and Research, Silver Spring, MD.

Gan J, Harper TW, Hsueh MM, Qu Q, and Humphreys WG (2005) Dansyl glutathione as a trapping agent for the quantitative estimation and identification of reactive metabolites. Chem Res Toxicol 18:896-903.

Gao H, Deng S, and Obach RS (2010) A simple liquid chromatography-tandem mass spectrometry method to determine relative plasma exposures of drug metabolites across species for metabolite safety assessments. Drug Metab Dispos 38:2147-2156.

Graham RA, Hop CE, Borin MT, Lum BL, Colburn D, Chang I, Shin YG, Malhi V, Low JA, and Dresser MJ (2012) Single and multiple dose intravenous and oral pharmacokinetics of the hedgehog pathway inhibitor vismodegib in healthy female subjects. Br J Clin Pharmacol 74: 788-796.

Hamilton RA, Garnett WR, and Kline BJ (1981) Determination of mean valproic acid serum level by assay of a single pooled sample. Clin Pharmacol Ther 29:408-413.

Harrison RK (2016) Phase II and phase III failures: 2013-2015. Nat Rev Drug Discov 15:817-818. Hastings KL, El-Hage J, Jacobs A, Leighton J, Morse D, and Osterberg RE (2003) Drug metabolites in safety testing. Toxicol Appl Pharmacol 190:91-92, author reply 93-94.

Hatsis P, Waters NJ, and Argikar UA (2017) Implications for metabolite quantification by mass spectrometry in the absence of authentic standards. Drug Metab Dispos 45:492-496.

Hop CE, Wang Z, Chen Q, and Kwei G (1998) Plasma-pooling methods to increase throughput for in vivo pharmacokinetic screening. J Pharm Sci 87:901-903.

International Conference on Harmonization (ICH) (2010) M3(R2) nonclinical safety studies for the conduct of human clinical trials and marketing authorization for pharmaceuticals.

International Conference on Harmonization (ICH) (2013) M3(R2) nonclinical safety studies for the conduct of human clinical trials and marketing authorization for pharmaceuticals. Questions and Answers(R2).

International Commission Radiological Protection (ICRP) (1991) 1990 recommendations of the international commission on radiological protection. ICRP Publication 60. Ann ICRP 21:1-201. International Commission on Radiological Protection (ICRP) (1992) Radiological protection in biomedical research. ICRP Publication 62. Ann ICRP 22.

International Commission on Radiological Protection (ICRP) ICRP (2007) The 2007 recommendations of the international commission on radiological protection. ICRP publication 103. Ann ICRP 37:1-332.

James AD, Marvalin C, Luneau A, Meissner A, and Camenisch G (2017) Comparison of ${ }^{19} \mathrm{~F}$ NMR and ${ }^{14} \mathrm{C}$ measurements for the assessment of ADME of BYL719 (alpelisib) in humans. Drug Metab Dispos 45:900-907.
Ku WW, Bigger A, Brambilla G, Glatt H, Gocke E, Guzzie PJ, Hakura A, Honma M, Martus HJ, Obach RS, et al.; Strategy Expert Group, IWGT (2007) Strategy for genotoxicity testingmetabolic considerations. Mutat Res 627:59-77.

Leclercq L, Cuyckens F, Mannens GS, de Vries R, Timmerman P, and Evans DC (2009) Which human metabolites have we MIST? Retrospective analysis, practical aspects, and perspectives for metabolite identification and quantification in pharmaceutical development. Chem Res Toxicol 22:280-293.

Li AC, Ding J, Jiang X, and Denissen J (2009) Two-injection workflow for a liquid chromatography/ LTQ-Orbitrap system to complete in vivo biotransformation characterization: demonstration with buspirone metabolite identification. Rapid Commun Mass Spectrom 23:3003-3012.

Lindon JC and Wilson ID (2015) 19F NMR spectroscopy: applications in pharmaceutical studies. eMagRes 4:189-196.

Luffer-Atlas D and Atrakchi A (2017) A decade of drug metabolite safety testing: industry and regulatory shared learning. Expert Opin Drug Metab Toxicol 13:897-900.

Ma S and Chowdhury SK (2011) Analytical strategies for assessment of human metabolites in preclinical safety testing. Anal Chem 83:5028-5036.

Ma S, Li Z, Lee KJ, and Chowdhury SK (2010) Determination of exposure multiples of human metabolites for MIST assessment in preclinical safety species without using reference standards or radiolabeled compounds. Chem Res Toxicol 23:1871-1873.

Morcos PN, Yu L, Bogman K, Sato M, Katsuki H, Kawashima K, Moore DJ, Whayman M, Nieforth K, Heinig K, et al. (2017) Absorption, distribution, metabolism and excretion (ADME) of the ALK inhibitor alectinib: results from an absolute bioavailability and mass balance study in healthy subjects. Xenobiotica 47:217-229.

Nedderman AN, Dear GJ, North S, Obach RS, and Higton D (2011) From definition to implementation: a cross-industry perspective of past, current and future MIST strategies. Xenobiotica 41:605-622.

Perkins C, Siddiqui S, Puri M, and Demain AL (2016) Biotechnological applications of microbial bioconversions. Crit Rev Biotechnol 36:1050-1065.

Pervaiz I, Ahmad S, Madni MA, Ahmad H, and Khaliq FH (2013) Microbial biotransformation: a tool for drug designing (Review). Prikl Biokhim Mikrobiol 49:435-449.

Prakash C, Li Z, Orlandi C, and Klunk L (2012) Assessment of exposure of metabolites in preclinical species and humans at steady state from the single-dose radiolabeled absorption, distribution, metabolism, and excretion studies: a case study. Drug Metab Dispos 40:1308-1320.

Prakash C, Sharma R, Gleave M, and Nedderman A (2008) In vitro screening techniques for reactive metabolites for minimizing bioactivation potential in drug discovery. Curr Drug Metab 9:952-964.

Reese MJ, Wurm RM, Muir KT, Generaux GT, St John-Williams L, and McConn DJ (2008) An in vitro mechanistic study to elucidate the desipramine/bupropion clinical drug-drug interaction. Drug Metab Dispos 36:1198-1201.

Rowland Yeo K, Jamei M, Yang J, Tucker GT, and Rostami-Hodjegan A (2010) Physiologically based mechanistic modelling to predict complex drug-drug interactions involving simultaneous competitive and time-dependent enzyme inhibition by parent compound and its metabolite in both liver and gut - the effect of diltiazem on the time-course of exposure to triazolam. Eur J Pharm Sci 39:298-309.

Ruan Q, Peterman S, Szewc MA, Ma L, Cui D, Humphreys WG, and Zhu M (2008) An integrated method for metabolite detection and identification using a linear ion trap/Orbitrap mass spectrometer and multiple data processing techniques: application to indinavir metabolite detection. $J$ Mass Spectrom 43:251-261.

Shitara Y, Hirano M, Sato H, and Sugiyama Y (2004) Gemfibrozil and its glucuronide inhibit the organic anion transporting polypeptide 2 (OATP2/OATP1B1:SLC21A6)-mediated hepatic uptake and CYP2C8-mediated metabolism of cerivastatin: analysis of the mechanism of the clinically relevant drug-drug interaction between cerivastatin and gemfibrozil. $J$ Pharmacol Exp Ther 311:228-236.

Smith DA and Dalvie D (2012) Why do metabolites circulate? Xenobiotica 42:107-126.

Smith DA and Obach RS (2005) Seeing through the mist: abundance versus percentage. Commentary on metabolites in safety testing. Drug Metab Dispos 33:1409-1417.

Smith DA and Obach RS (2006) Metabolites and safety: what are the concerns, and how should we address them? Chem Res Toxicol 19:1570-1579.

Smith DA and Obach RS (2009) Metabolites in safety testing (MIST): considerations of mechanisms of toxicity with dose, abundance, and duration of treatment. Chem Res Toxicol 22:267-279.

Staffa JA, Chang J, and Green L (2002) Cerivastatin and reports of fatal rhabdomyolysis. $N$ Engl J Med 346:539-540.

Swart P, Frederic Lozac'h F, and Zollinger M (2016) A Mass Balance and Metabolite Profiling Study of Sonidigib in Healthy Male Subjects Using Microtrace Approach, John Wiley \& Sons Inc., Hoboken, NJ.

Synal HA, Stocker M, and Suter M (2007) MICADAS: a new compact radiocarbon AMS system. Nucl Instrum Meth B 259:7-13.

Takahashi RH, Khojasteh C, Wright M, Hop CECA, and Ma S (2017) Mixed matrix method provides a reliable metabolite exposure comparison for assessment of Metabolites in Safety Testing (MIST). Drug Metab Lett 11:21-28.

Taub ME, Ludwig-Schwellinger E, Ishiguro N, Kishimoto W, $\mathrm{Yu} \mathrm{H}$, Wagner $\mathrm{K}$, and Tweedie D (2015) Sex-, species-, and tissue-specific metabolism of empagliflozin in male mouse kidney forms an unstable hemiacetal metabolite (M466/2) that degrades to 4-hydroxycrotonaldehyde, a reactive and cytotoxic species. Chem Res Toxicol 28:103-115.

Tong Z, Chandrasekaran A, DeMaio W, Espina R, Lu W, Jordan R, and Scatina J (2010a) Metabolism of vabicaserin in mice, rats, dogs, monkeys, and humans. Drug Metab Dispos 38: 2266-2277.

Tong Z, Chandrasekaran A, DeMaio W, Jordan R, Li H, Moore R, Poola N, Burghart P, Hultin T, and Scatina J (2010b) Species differences in the formation of vabicaserin carbamoyl glucuronide. Drug Metab Dispos 38:581-590.

Trosset JY and Carbonell P (2015) Synthetic biology for pharmaceutical drug discovery. Drug Des Devel Ther 9:6285-6302.

van Duijn E, Sandman H, Grossouw D, Mocking JA, Coulier L, and Vaes WH (2014) Automated combustion accelerator mass spectrometry for the analysis of biomedical samples in the low attomole range. Anal Chem 86:7635-7641.

Vuong LT, Song Q, Lee HJ, Roffel AF, Shin SH, Shin YG, and Dueker SR (2015) Opportunities in low-level radiocarbon microtracing: applications and new technology. Future Sci OA 2:FSO74

Walker D, Brady J, Dalvie D, Davis J, Dowty M, Duncan JN, Nedderman A, Obach RS, and Wright P (2009) A holistic strategy for characterizing the safety of metabolites through drug discovery and development. Chem Res Toxicol 22:1653-1662. 
Walker GS, Bauman JN, Ryder TF, Smith EB, Spracklin DK, and Obach RS (2014) Biosynthesis of drug metabolites and quantitation using NMR spectroscopy for use in pharmacologic and drug metabolism studies. Drug Metab Dispos 42:1627-1639.

Walker GS and O'Connell TN (2008) Comparison of LC-NMR and conventional NMR for structure elucidation in drug metabolism studies. Expert Opin Drug Metab Toxicol 4:1295-1305.

Webster GK and Kumar S (2014) Expanding the analytical toolbox: pharmaceutical application of quantitative NMR. Anal Chem 86:11474-11480.

Williams AJ, Martin GE, and Rovnyak D (2016) Modern NMR Approaches to the Structure Elucidation of Natural Products, Royal Society of Chemistry, Cambridge, UK

Xu L, Woodward C, Dai J, and Prakash C (2013) Metabolism and excretion of 6-chloro-9-(4methoxy-3,5-dimethylpyridin-2-ylmethyl)-9H-purin-2-ylamine, an HSP90 inhibitor, in rats and dogs and assessment of its metabolic profile in plasma of humans. Drug Metab Dispos 41 2133-2147.

Yeung CK, Fujioka Y, Hachad H, Levy RH, and Isoherranen N (2011) Are circulating metabolites important in drug-drug interactions? quantitative analysis of risk prediction and inhibitory potency. Clin Pharmacol Ther 89:105-113.

Yu C, Chen CL, Gorycki FL, and Neiss TG (2007) A rapid method for quantitatively estimating metabolites in human plasma in the absence of synthetic standards using a combination of liquid chromatography/mass spectrometry and radiometric detection. Rapid Commun Mass Spectrom 21:497-502.
Yu H, Balani SK, Chen W, Cui D, He L, Humphreys WG, Mao J, Lai WG, Lee AJ, Lim HK, et al. (2015) Contribution of metabolites to P450 inhibition-based drug-drug interactions: scholarship from the drug metabolism leadership group of the innovation and quality consortium metabolite group. Drug Metab Dispos 43:620-630.

Yu $\mathrm{H}$ and Tweedie D (2013) A perspective on the contribution of metabolites to drug-drug interaction potential: the need to consider both circulating levels and inhibition potency. Drug Metab Dispos 41:536-540.

Zhu M, Ma L, Zhang D, Ray K, Zhao W, Humphreys WG, Skiles G, Sanders M, and Zhang H (2006) Detection and characterization of metabolites in biological matrices using mass defect filtering of liquid chromatography/high resolution mass spectrometry data. Drug Metab Dispos 34:1722-1733.

Zhu M, Zhang H, and Humphreys WG (2011) Drug metabolite profiling and identification by highresolution mass spectrometry. J Biol Chem 286:25419-25425.

Address correspondence to: Simone Schadt, Roche Pharma Research and Early Development, Pharmaceutical Sciences, Roche Innovation Center Basel, F. Hoffmann-La Roche Ltd., Grenzacher Strasse 124, 4070 Basel, Switzerland. E-mail: simone.schadt@roche.com 